\title{
Prescribing Mindfulness for Heart Health
}

\author{
Masoud Ghaffari* \\ Department of Nursing, Benedictine University, USA.
}

*Corresponding author: Masoud Ghaffari, Department of Nursing, Benedictine University, USA.

Received Date: September 05, 2018

Published Date: September 13, 2019

\begin{abstract}
Scientific research has increasingly investigated "alternative" and "complementary" (CAM) therapies such as meditation for a variety of challenging psychological and physiological human conditions in the last few decades. The use of meditation for healing and enlightenment is not new. The practice of meditation has been prevailing throughout the human history among diverse cultures. In fact, all religious traditions practice some forms of meditation. Meditation is a mental training capable of producing connection between the mind, body and spirit. Research studies on the biological and clinical benefits of mindfulness meditation are providing increasing evidence about the short- and long-term changes that occur in mindfulness meditators and about clinical outcomes in physically ill, mentally ill, as well as in healthy subjects related to such practices. Cardiovascular disease (CVD) remains the leading cause of death in the United States as well as one of the most expensive to the health care system. The Centers for Disease Control and Prevention estimated about 20\% of total healthcare costs is spent on CVD treatments. Mind/body techniques are a commonly used category of CAM in people with hypertension. Mind/body techniques include meditation and moving meditations such as yoga, Tai Chi, and Qi Gong. Patients claim these techniques increase their health and well-being. The aim of this article is to review the existing literature in order to explore and describe what meditation is, its practices and effects on health, demonstrated by consistent scientific investigations.
\end{abstract}

Keywords: Cardiovascular disease; Coronary artery disease; Meditation; Mind-body therapies; Integrative treatment; Meditation; Spirituality; Complementary therapy

\section{Introduction}

Complementary and alternative medicine (CAM) consists of a wide range of practices that are not part of allopathic, or conventional medicine. Generally, this is because there is insufficient evidence to support the safety and efficacy of these treatments. However, evidence is mounting to support the use of many CAM methods [2]. Scientific research has increasingly investigated "complementary" therapies such as meditation for a variety of challenging human conditions in the last few decades. While the medical community has caught on to the physical and emotional health benefits of meditation, religious and spiritual scholars have long known the benefits of faith-based practices on health and wellness [1].

The use of CAM practices is prevalent in the United States. A study conducted by the National Institutes of Health (NIH) found that in 2007, $40 \%$ of adults in the United States reported using CAM practices within the last 12 months. The most common type of CAM treatments were natural products (non-vitamin, nonmineral) and deep breathing exercises. There was growth in the use of acupuncture, naturopathy, message therapy, deep breathing, exercise, yoga and meditation. Furthermore, many adults reported utilizing CAM treatments when the cost of conventional treatments was a concern [2].

The use of yoga with deep breathing and meditation are common CAM treatments in the United States. Data from the 2012 National Health Interview Survey found that on average $8.4 \%$ of Americans had participated in yoga with deep breathing or meditation within the last year [3]. The highest use of these CAM treatments was found in the Pacific region (12.1\%) and the lowest use was in the East South-Central region (5.1\%) [3]. Because CAM treatments, including deep breathing and meditation are relatively common in the United States, it is important for health care providers to have knowledge of how they are practiced and what their potential benefits are.

There are several types of meditative practice, originating from different CAM systems. Mindfulness meditation (MM) is a contemporary form of meditation that stems from Buddhist traditions. Studies on the benefits of MM have demonstrated numerous benefits, including improving cardiovascular health. Mindfulness meditation is associated with lowered cardiovascular 
disease risk and may have cardio-protective effects through modulation of the autonomic nervous system and the inflammatory response. This supports the importance of the mind-body connection (psychoneuroimmunology) in relation to health. Furthermore, MM is a helpful tool that medical providers can recommend to patients decrease stress, anxiety, depression, cognitive decline and pain, as well as improve cardiovascular health. The aim of the present work is to review current evidence about the effects of meditation in general, and specifically on cardiovascular health. The author also aims to provide an integration of these data in order to address future research on the topic.

\section{Literature Review}

Initially, relevant texts were identified by general internet searches using Google Scholar. Then, a literature search was undertaken using CINHAL, PubMed, the Cochrane database, ProQuest Central, and references of retrieved articles. The search was restricted to full text publications and those published only in English. The main search terms were mindfulness meditation, Zen meditation, Mindfulness-Based Stress Reduction, MindfulnessBased Cognitive Therapy, meditation and CVD, and CAM therapies for CVD. The main reason for exclusion was non-research-based articles. The author scanned all potentially relevant articles published between 1966 and 2018 to examine the literature on mindfulness meditation and its effects on cardiovascular diseases. The first empirical paper on mindfulness meditation was appeared in 1966.

\section{What Is Meditation?}

Meditation is a method of achieving relaxation and calming the mind. Americans are often bombarded with stimuli and face internal and external pressure to stay busy and accomplish as much as possible during each day. Meditation is a way of calming the endless mental activity and thoughts. It is thought that meditation can help realign the mind and body and help bring the individual back into balance. Meditation has been used for thousands of years beginning in ancient health care traditions, but in the last 30 years, it has also become popular in Western culture [4].

The word meditation in Sanskrit comes from the word 'dhyana' which means attention, contemplation. The word "meditation" is derived from the Latin meditari, which means "to engage in contemplation or reflection." The word meditation comes from the same Greek and Latin root as the word medicine. The exercise of meditation is processed by a large variety of activities that range from techniques to promote relaxation through to exercises performed with objectives that are broader in scope, such as intensification of the feeling of wellbeing [5]. It also includes breathing techniques, repetition of sounds and/or observation of the process of thought to focus attention and promote a state of self-awareness and inner calm [6]. In the East, meditation is an ancient practice, developed to broaden consciousness and seek health. Ancient texts, with reference to the Indian Ayurvedic system of medicine, demonstrate that this practice formed part of medical procedures used for the recovery and maintenance of health that go back further than 3000 years ago Sampaio CVS , et al. [7], whereas, in the West, the tradition of meditation can be linked to religious experiences, especially Christian with a Catholic or Protestant connotation. On the other hand, meditation is typically pursued in Western nations as a secular practice oriented around contemplation and mindfulness. With its largely secular, therapeutic, and pragmatic focus, Western meditation is quite different from that associated with traditional Buddhism and Eastern mysticism. As practiced in the West, meditation is a broad set of practices that can be categorized as a complementary approach to health, and can include yoga, tai chi, and qi gong [8].

As was mentioned earlier, Meditation has been used for more than a thousand years as a spiritual or healing practice. It encompasses a set of practices that can be subdivided into three umbrella groups: 1) attentional (as in mindfulness meditation), 2) constructive (as in compassion or loving kindness meditation), and 3) deconstructive (as in object-oriented insight) [9]. In the past 20 years, in Western societies, there has been an increase in the use of meditation practices related to mindfulness-based interventions (MBI), one of the most frequent meditative approaches used in the scientific context.

To understand the process of meditation better, Lauricella $\mathrm{S}$ et al. [10] presents the following analogy:

Several times a day, most of us throw trash in a receptacle, morning and night we brush our teeth, and once a day most of us will have a bath or shower. We launder and press our clothes every few days, take the recycling to the curb weekly, and get our vehicles serviced quarterly inconveniences. However, stress is the plight of the modern human, and health issues such as high blood pressure, insomnia, and headaches are part of its consequences, particularly in the West. Yet many of us do very little to contribute positively to our mental or spiritual hygiene. One way in which more and more people, particularly in the West, are committing to improve their mental and spiritual wellbeing is via practicing meditation (or at least we should). We do these tasks with the objective to foster positive experiences in relationships, achieve a better state of physical health, and to experience less (p. 1748).

\section{The Science of Meditation}

For the majority of researchers, there are two general types of meditation: 1) concentration meditation and 2) mindfulness meditation [5]. The former emphasizes the need for attention focused on an object, and to sustain this process until the mind attains quieting of thoughts. The initial researches with meditation investigated its effects on human physiology [11]. This study found that during meditation, a reduction occurs in oxygen consumption and cardiac frequency, and an increase in galvanic resistance of the skin. Furthermore, the electroencephalogram showed a predominance of alpha waves, thus it was concluded that these physiological alterations were compatible with changes in autonomic activity, indicative of reduction in sympathetic activities, and therefore, meditation could have applications in clinical medicine [11]. 
There are different techniques for meditation that originate from different CAM disciplines. In the past, meditation was used primarily for religious or spiritual growth. Many meditative traditions originate from India, Tibet, China and Japan, but meditation practices are part of CAM systems around the world. As I mentioned earlier, more modern meditative practices in the United States are secular in nature and are often used to decrease stress and improve mental and physical health [4].

The use of meditation for healing and enlightenment is not new. However, it was only during the 1960s that scientific studies started focusing on the clinical effects of meditation on health. With the scientific advancement and refinement in instrumentation, scientific study of effects of meditative practices became possible. A formal acknowledgement of the academic curiosity within psychology came in 1977, when the American Psychological Association (APA) issued a statement on meditation stating that"meditation may facilitate the psychotherapeutic process". Hussain D et al. [12] described meditation as a discrete and well-defined experience of a state of "thoughtless awareness" or mental silence, in which the activity of the mind is minimized without reducing the level of alertness [12].

Thus, meditation has been conceptualized in many ways and there is no consensus definition. It is very difficult to capture its essence in one definition. However, Hussain D et al. [12] developed an operational definition encompassing both traditional and clinical parameters. They defined any practice as meditation if it (1) utilizes a specific and clearly defined technique, (2) involves muscle relaxation somewhere during the process, (3) involves logic relaxation (i.e., not "to intend" to analyze the possible psychophysical effects, not "to intend" to judge the possible results, not "to intend" to create any type of expectation regarding the process), (4) a self-induced state, and (5) the use of a self-focus skill or "anchor" for attention [12].

Heart rate-Studies have indicated that heart rate slows down during quite meditation and quickens in the moments of ecstasy during meditation. Meditations like TM, Zen, relaxation response and other calming forms of meditation generally decrease the rate of heartbeat. However, very pronounced decrease in heart rate is found among long term practitioners only.

Blood pressure and hypertension-Blood pressure is one of the easiest measurable physiological variables. There is strong evidence that meditation lowers blood pressure for the people who are normal or moderate hypertensive. However, most studies indicate that the benefit disappears once practice is discontinued.

Cortical activity—Evidence from many studies indicates that during meditation alpha activity increases significantly. Alpha waves are slow and high amplitude brain waves with frequency ranging from eight to thirteen cycles per second.

Helminiak DA et al. [13] described six possible mechanisms through which meditation works. These six mechanisms capture most of the explanations found in the existing literature. They are:

\section{Relaxation}

Relaxation is one of the primary components of all kinds of meditation which induce a pleasant and deep relaxed state of body and mind. Herbert Benson (1976) developed a therapeutic technique called "relaxation response" which is a form of meditation. His whole concern with meditation was to reduce stress and hypertension by inducing a state of deep relaxation. Benson measured series of physiological parameters in response to relaxation response. Various effects include-decrease in the rate of metabolism, decrease in the rate of heartbeat, muscle relaxation, slow and rhythmic breathing, decrease in blood pressure, and so on. All these effects help in balancing physiological abnormalities and promotes healing.

\section{Systematic desensitization}

Wolpe J et al. [14] behavioral therapy is especially instrumental in reducing anxiety. This therapy involves three steps. First, the client is thought to induce a deep state of muscle relaxation. This is followed by preparing a hierarchical list of stimuli inducing anxiety. Finally, in a deep relaxed state client confronts (either by imagination or by presentation of actual stimuli) each of the anxiety producing stimuli progressing in hierarchy. This therapy is based on the principle of reciprocal inhibition. Since anxiety and relaxation are incompatible to each other, the stimuli lose their anxiety provoking quality. Client continues this process until he is desensitized to highest item in the hierarchy. In meditation also a practitioner undergoes similar steps.

\section{Release of repressed psychic material}

This is related to systematic desensitization. With regular practice of meditation, most of the practitioners encounter release of repressed unconscious thoughts, emotions, and images [15]. During meditation, the practitioner remains under low arousal and sensory deprivation for a long time and under such condition repressed feelings and thoughts arises.

\section{Un-stressing}

Parallel to release of repressed memories, many practitioners reports many physical reactions during intense meditation. This could be involuntary muscular-skeletal movements such as repeated twitches, spasms, gasps, tingling, tics, jerking, swaying, pains, shaking, aches, internal pressures, headaches, weeping, and laughter. The experience covers the range from extreme pleasure to acute distress. TM practitioner calls this as "unstressing". Goleman D et al. [16] interprets this phenomenon on the basis of psycho-physiological principle contemplating that all psychic and emotional phenomena have parallel physiological processes.

\section{Dissolution of habitual patterns of perception}

Human beings are mostly governed by rigid and fixed patterns of thinking, feeling, and reactions. Many of these patterns are unhealthy and cause neurotic and psychotic problems. Most of the unhealthy habitual patterns are due to our identification with emotions that we are not able to control and regulate. With detached observation, emotions and thoughts lose their power and 
practitioner can identify the unhealthy patterns of behavior and remove them with healthy ones.

\section{Cosmic consciousness}

Attainment of cosmic consciousness is a mystical concept and not available for scientific investigation. Many terms are used to represent cosmic consciousness such as samadhi, nirvana, satori, and moksha. It is the highest goal achieved by meditation in esoteric traditions where a person transcends his personal ego. In the state of cosmic consciousness, a person realizes that he/ she is one with the whole cosmos and is not separate from others. As a result, a tremendous sense of love and compassion arises in him and it is the highest state a human can achieve [cited in 12].

\section{Types of Meditation}

There are several different methods of meditation. Meditation can be contemplative or active and it may or may not have a spiritual component. Several ancient medical systems employ meditation as a health maintenance practice. The most common forms of mediation are Tai Chi from Traditional Chinese Medicine (TCM), Yoga from the Ayurveda CAM system and contemporary meditation including Transcendental Meditation and Mindfulness Meditation or MM [17].

\section{Traditional chinese medicine and T'ai Chi}

T'ai Chi is an active form of meditation that originated from TCM. It has been used for many years in China but has recently become popular in the United States [4]. T'ai Chi (also known as Tai Chi Chuan or Taijiquan) is a martial art that emphasizes body movement, breath and meditation. T'ai Chi practice involves slow, continuous flow of movement from one position to the next, and promotes good posture, balance and mental concentration [18].

In TCM, T'ai Chi is considered a form of Qigong. Qigong is a term used to describe methods to cultivate qi, or the universal life force. It is a type of energy medicine that is thought to improve overall health and facilitate healing by strengthening the individual's energy system. Internal Qigong is a self-directed practice that is thought to improve health by restoring qi. It involves exercises including T'ai Chi and other martial arts, as well as breathing and meditation exercises [19]. Researchers have identified several benefits to T'ai Chi, including, preserving the cognitive abilities of older adults, relieving anxiety and depression, preventing falls, and improving immune function $[4,18,20,21]$.

\section{Ayurveda and yoga}

Yoga is another active method of meditation. It is part of the Ayurveda CAM system that originated in India. Like TCM, Ayurveda focuses on energy systems and the promotion of correct flow of energy. While many yoga practitioners in the United States focus on physical movements, stretching and balancing, traditional Ayurvedic yoga is much more than just physical movements and poses. In fact, there are eight limbs of yoga, which are: moral and ethical discipline, restraint, posture, breath control, sensory inhibition, concentration, meditation and ecstasy. Each of the eight limbs is a step toward self-transcendence [22].
Meditation is an essential component of most yoga practices, including contemporary yoga practiced in the United States. In yoga, meditation is preceded by concentration, which prepares the mind for meditation. Within yoga practices, there are different styles of meditation. Focused attention yoga involves focusing the mind on one item, while open monitoring meditation involves focusing on the present moment without focusing on any single item. Furthermore, use of a mantra is also common in yoga meditation. A mantra is a word or series of words that are chanted during meditation. According to traditional yoga beliefs, a mantra stimulates energy pathways due to the vibration and meaning of the words. Another proposed reason for the benefit of using a mantra is that the breath is slowed during the recitation of a mantra, which leads to decreased blood pressure [22].

Many benefits of yoga practice have been identified, including in treating pain conditions, cancer, cardiovascular disease, mental health conditions and diabetes [22]. Yoga has also been shown to decrease inflammatory markers [23]. Furthermore, several benefits during pregnancy have been identified, including improved mental health, reduced pelvic pain and improved perinatal outcomes such as decreased complications and delivery time [24].

\section{Transcendental meditation}

Contemporary meditation practices have roots in the traditional CAM systems but have been adapted to Western culture and society. Transcendental meditation (TM) was introduced in the United States in the 1960s by Maharishi Mahesh Yogi who was from India. To make TM more palatable to Western society, he changed traditional yogic meditation and eliminated the more difficult elements. In TM, a practitioner sits comfortably and repeats a specific mantra silently, focusing entirely on the mantra. When distractions come into the mind, they are to be noted and then attention refocused on the mantra. Transcendental meditation is generally practiced for 15 to 20 minutes in the morning and in the evening [4]. Although it is not a religious practice, transcendental meditation has a spiritual component. According to its teachings, as practitioners focus on the sound of the mantra, they can transcend the physical world [17].

Transcendental meditation has been shown to be very helpful in managing anxiety. In a meta-analysis of randomized controlled trials (RTCs), Orme-Johnson and Barnes [25] determined that TM is more effective than standard medical treatment and other alternative treatments for anxiety. Furthermore, Wachholtz AB et al. [26] conducted an RTC examining spiritual meditation with secular meditation and relaxation and found that practitioners of spiritual meditation (including TM) had greater decrease in anxiety, more positive mood and were able to endure more pain than either the secular meditation group or the relaxation group. Other benefits of TM include treatment of substance abuse, reduction in blood pressure, and reduction in other cardiac risk factors [4].

\section{Mindfulness meditation}

Mindfulness meditation is based on traditional Buddhist meditation techniques that were used at least 2,500 years ago. It has 
recently been adapted for use in reducing stress and treating other conditions in Americans. Jon Kabat-Zinn started the mindfulness movement in the 1990s to help patients with pain and stress-related disorders. While the concept of mindfulness began in Buddhist traditions, it has undergone many changes in its adaption for use in the United States. In the Buddhist tradition, meditation is one piece in an integrated system that is designed to free individuals from suffering. Other aspects include living an ethical life, studying the teachings of Buddha, and adhering to other spiritual and religious practices. Furthermore, Buddhist meditation focuses the mind on the non-self and impermanence of thoughts, feelings and bodily discomfort because understanding the impermanence of things and being detached from a fixed idea of self leads to detachment from suffering. Western MM is somewhat simplified and focuses on nonjudgmental observation of internal and external occurrences moment-to-moment [27].

The process of mindfulness meditation involves focusing the attention on one point. As thoughts and feelings arise, they are not suppressed or judged, but rather observed and accepted. By observing them without judgment, the person can become detached from them, which provides a broader perspective. Negative feelings should be noted and accepted because accepting those feelings is the first step releasing the impact that they have on a person's life. Mindfulness meditation is not designed to remove pain and stress from an individual's life. Rather it is a practice that helps people focus more clearly and respond to stress and pain more effectively [4]. Mindfulness has the ability to help regulate attention as it is focused on what is happening in the current moment. It also helps individuals approach experiences with openness and acceptance. Overall, mindfulness leads to enhanced attention control, emotional regulation and self-awareness, which all contribute to self-regulation.

Mindfulness can combat the common tendencies that lead to psychological distress, such as anxiety, worry, fear, and anger and it helps individuals avoid maladaptive strategies for dealing with negative feelings, such as avoidance, suppression or obsession with them [27]. In the early stages of MM training, individuals practice focused attention on a single object or idea. During this time, the monitoring ability of the brain is utilized for redirecting thoughts when the mind wanders. In more advanced practitioners, focus on a specific object is reduced, and open monitoring meditation is used [28].

Mindfulness meditation can improve focus. Garrison KA et al. [29] found that individuals who practice MM have decreased default mode network (DMN) activity while performing activities. Default mode network activity is related to mind wandering and self-related thinking. Individuals with decreased DMN experience improved sustained attention. In this study, control subjects and meditators were asked to perform cognitive tasks while undergoing functional magnetic resonance imaging (fMRI). The results demonstrated decreased activity in areas associated with DMN, which are the posterior cingulate/precuneus and the anterior cingulate cortex. Similarly, in a systematic review, Chiesa A et al. [28] found that in the early phases of MM training, individuals show significant improvement in selective and executive attention. In later stages of training, subjects have improved sustained attention abilities.

In a systematic review of brain imaging studies conducted using MM, Tang Y et al. [30] determined that several areas of the brain demonstrate changes in meditators compared to controls. They consistently found changes in the anterior cingulate cortex, which is associated with executive attention and control. They also noted changes in the dorsolateral prefrontal cortex, which is associated with executive processing. Changes in the brain in areas related to emotional responses were also identified, including prefrontal regions, limbic regions and the amygdala. These authors also determined that there were significant differences in experienced meditators, compared to novice meditators. For example, novice meditators had increased activity in prefrontal regions, as compared to experts. This supports the idea that novice meditators utilize the prefrontal region to actively control their thoughts and emotions, while experts have automated this process [30].

Mindfulness meditation has also been found to slow cognitive decline in the elderly. Gard $\mathrm{T}$ et al. [31] conducted a systematic review and found that, although most studies reviewed had small sample sizes and a high risk for bias, the evidence suggests that MM has a positive impact on executive function, attention, memory and general cognition in the elderly. This was also supported by neuroimaging studies that found that older meditators had less age-related decrease in grey matter volume within the putamen, which is an area in the brain associated with age-related decline in sustained attention [30].

\section{Programs Based on Mindfulness Meditation}

Several programs have been developed based on MM. These include mindfulness-based stress reduction (MBSR), mindfulnessbased cognitive therapy (MBCT), and dialectical behavior therapy (DBT). In MBSR, group sessions offer intensive MM training as an adjunct treatment for chronic pain. Participants learn to relate to their physiological conditions in accepting and non-judgmental ways. MBSR also incorporates mindful yoga and gentle stretching. It is generally an eight to ten-week program. Group sessions are two and a half hours per week, and participants are encouraged to practice MM individually at home, as well as to attend a full day MM retreat during the program $[27,32]$.

Mindfulness-based cognitive therapy is a variation of cognitive behavioral therapy in which participants learn to change their awareness and relationship with their thoughts and emotions through MM. It is an eight-week group-based program that was designed to treat patients with depression who repeatedly experience relapse [27]. Dialectical Behavior Therapy incorporates cognitive behavioral therapy with Buddhist philosophy in the treatment of recurrent suicidal thoughts and self-harm in patients with borderline personality disorder and other psychological conditions. It teaches MM as a skill that helps patients cope with their intrusive thoughts and feelings that lead to self-harm. Multiple studies have demonstrated that mindfulness-based therapies are 
beneficial for several psychological and pain-related conditions [27].

Mindfulness-based stress reduction is also beneficial for healthy individuals. In a systematic review, Sharma M et al. [33] found that MBSR improved stress and anxiety levels in 16 out of 17 studies reviewed involving healthy participants. Similarly, MBSR is helpful for health care professionals for reducing burnout, stress, anxiety and depression, which was found in a systematic review of 39 studies [34]. Mindfulness meditation has benefits for psychological stress and well-being. In a systematic review and meta-analysis of 47 studies with 3,320 participants, Goyal M et al. [35] found strong evidence that MM programs decreased anxiety, depression and pain. They found weaker evidence that MM decreases stress and improves mental health-related quality of life.

\section{Cardiovascular Benefits}

An important area of research related to MM is its effect on cardiovascular health. Heart disease, including coronary artery disease, myocardial infarction, angina, aortic aneurysm and dissection, arrhythmias and atrial fibrillation, cardiomyopathy, heart failure, congenital heart defects, peripheral arterial disease and rheumatic heart disease, is a major cause of morbidity and mortality in the United States. Heart disease is the number one cause of death in the United States for both men and women. In the U.S., the total cost of health care services, medications, and lost productivity due to heart disease is $\$ 108.9$ billion each year [36].

Several studies have confirmed a cardiovascular benefit from MM. A systematic review and meta-analysis of 11 RTCs revealed that MM improves several markers for cardiovascular health, including physical quality of life, mental quality of life, depression, anxiety, systolic blood pressure and diastolic blood pressure Younge JO et al. [37] Similarly, Loucks EB et al. [32] found that mindfulness has a positive impact on cardiovascular risk factors, including physical activity, smoking, diet, obesity, lipids, diabetes and blood sugar control, and blood pressure. The authors postulated that this is due to the enhanced self-regulation that MM cultivates which leads to improved attention control, emotional regulation and selfawareness.

Dispositional mindfulness is a modifiable trait that defines the degree to which an individual can attend to the present moment. In a cross-sectional study examining the relationship between mindfulness disposition and cardiovascular health, Loucks EB et al. [38] found that individuals with dispositional mindfulness had improved cardiovascular health. This association was primarily driven by associations between mindfulness and cardiovascular risk factors, including smoking, diabetes, physical activity, and body mass index.

Even a brief MM intervention can improve cardiovascular health-related variables. College students exposed to a total of one-hour MM training over three days demonstrated a reduction in negative mood, depression, fatigue, confusion, and heart rate compared to control subjects who received sham MM instructions [39]. In a quasi-experimental, pre-test, post-test study, May R et al.
[40] provided subjects with a 15-minute MM exercise and found that they had improved cardiovascular autonomic regulation as measured by blood pressure variability and heart rate variability. Because MM allows for a wakeful hypometabolic state, sympathetic activation is limited, and parasympathetic tone dominates. This counteracts the harmful effects of overstimulation of the sympathetic nervous system, including increased cardiac workload, endothelium dysfunction, myocardial infarction and coronary spasm [41].

Another potential cardiovascular benefit of MM is inhibition of the inflammatory response, which is implicated in the progression of atherosclerosis and thrombus development [41]. In a recent meta-analysis, authors determined that mind-body techniques, such as yoga and meditation reduce markers of inflammation and can increase the immune response to vaccination [42]. This hypothesis is supported by a study conducted by Kaliman P et al. [43], who found decreased expression of proinflammatory genes in expert meditators after a day of intensive MM.

Factors such as decreased inflammation and sympathetic tone that are caused by MM signify a link between the brain and the heart, or a mind-body connection. There is further evidence of this in the fact that people with depression have much worse cardiovascular health than non-depressed individuals [41]. Gao W et al. [44] conducted a study using electroencephalogram (EEG) and electrocardiogram (ECG) measurements during MM. They found that during MM training, the chaotic activities of the brain and heart became more coordinated, leading them to make the conclusion that MM training caused entrainment of the mind and body.

\section{Conclusion}

The aim of the present work was to review and synthesize current evidence about MM. The author also aimed to provide an integration of these data in order to address future research on MM.

More and more Americans use CAM treatments for maintaining health and treating health conditions. Therefore, health care providers need to learn about different CAM methods and understand which CAM interventions are supported by research evidence. Meditative practices, including MM appear to be useful for several medical conditions and for improving overall health. Mindfulness meditation in particular appears to be helpful for improving cardiovascular health. The positive effects of meditation on physical health support the importance of understanding the mind-body connection. Health care providers need to understand and utilize this connection in caring for their patients and themselves.

Meditation is believed to be efficacious in reducing sympathetic activity, lowering cortisol levels via modulation of the hypothalamic pituitary adrenal pathway, and reducing negative behavioral activity. Studies have shown that in addition to decreasing CV mortality, the beneficial effects of meditation improve conditions such as hypertension, type 2 diabetes mellitus, dyslipidemia, and high cortisol levels. 
Most Western practitioners of Complementary and alternative (CAM) medicine recommend some type of meditative technique to achieve optimal mind-body health. While there are various techniques and schools of thought on the best way to achieve the benefits of this mind-body connection, many different cultures throughout the world have used these techniques for thousands of years and offer an opportunity to explore these differences and the results. Whether for religious, spiritual, cultural or health/wellness purposes or a combination of all of these, these Eastern techniques have established a long history of concentrating on this mindbody relationship to not just heal a person but to achieve optimal physical, spiritual, and mental health.

Patients with CV risk factors such as hypertension and type 2 diabetes mellitus would likely benefit from meditation. Prospective trials are needed to study the effects of meditation on $\mathrm{CV}$ risk factors such as lipid profile, C-reactive protein, type 2 diabetes mellitus, and hypertension. Future studies are also needed to provide guidelines (duration, frequency) for daily use of meditation in everyday life.

In the 2007 review of Meditation Practices conducted by the Agency for Healthcare Research and Quality, one common feature of all the meditation practices studied was the apparent ability to practice meditation without adopting a specific system of spiritual or religious belief [45]. However, the researchers were quick to point out that if Taoist metaphysical assumptions of Qi Gong are crucial to successfully understand, visualize, and guide qi, then at least this practice requires adopting a specific belief system [45]. Though the traditional practices were developed within specific spiritual or religious contexts (Vipassana, Zen Buddhism, Yoga, Tai Chi, Qi Gong), an individual practitioner does not have to have the same belief system in order to derive benefit from the exercise. Perhaps a pearl of wisdom from the Dalai Lama provides the best rationale for becoming a Practitioner of Meditation.

Meditation is the process of familiarizing ourselves with the virtues that bring about happiness. This enables us to embody those virtues and to clearly realize the profound truths that are hidden from us in our daily lives. It is a technique by which we diminish the force of old thoughts and habits and develop new ones. Gain control over the mind and guide it in a more virtuous direction [46].

\section{Acknowledgment}

None.

\section{Conflict of Interest}

No conflict of interest.

\section{References}

1. Kreps G (2012) The role of prayer in promoting health and wellbeing The Journal of Communication and Religion 35(3): 237-253.

2. Barnes PM Bloom B (2008) Complementary and alternative medicine use among adults and children. Natl Health Stat Report (12): 1-23.

3. Peregoy JA, Clarke TC, Jones LI, Stussman BJ, Nahin, RL (2014) Regional variation in use of complementary health approaches by U.S. adults. NCHS Data Brief (146): 1-8.
4. Micozzi MS, Jawer MA (2015) Mind-body therapies, stress and psychometrics. In: Micozzi MS (Ed), Fundamentals of Complementary and Alternative Medicine, pp. 114-140.

5. Lutz A, Slagter HA, Dunne JD, Davidson RJ (2008) Attention regulation and monitoring in meditation. Trends Cogn Sci 12(4): 163-169.

6. Canter P (2003) The therapeutic effects of meditation. BMJ 326(7398): 1049-1050.

7. Sampaio CVS, Lima MG, Ladeia AM (2017) Meditation, health and scientific investigations: Review of the literature. J Relig Health 56(2): 411-427.

8. Clarke LI, Black BJ, Stussman PM, Barnes, Nahin RL (2015) Trends in the Use of Complementary Health Approaches among Adults: United States, 2002-2012. Natl Health Stat Report (79): 1-6.

9. Dahl CJ, Lutz A, Davidson RJ (2015) Reconstructing and deconstructing the self: cognitive mechanisms in meditation practice. Trends Cogn Sci 19(9): 515-538

10. Lauricella S (2016) The ancient-turned-new concept of "spiritual hygiene": An investigation of media coverage of meditation from 1979 to 2014. J Relig Health 55(5): 1748-1762.

11. Wallace R (1970) Physiological effects of transcendental meditation. Science 167(3926): 1751-1754.

12. Hussain D, Bhushan B (2010) Psychology of meditation and health: Present status and future directions. International Journal of Psychology and Psychological Therapy 10(3): 431-451.

13. Helminiak DA (1981) Meditation-Psychologically and Theologically Considered. Pastoral Psychology 30(1): 6-20.

14. Wolpe J (1961) The Systematic Desensitization Treatment of Neuroses. The Journal of Nervous and Mental Disease 132(3): 189-203.

15. Schwartz GE (1974) TM Relaxes Some People and Makes Them Feel Better. Psychology Today April: 39-44.

16. Goleman D (1976) Meditation and Consciousness: An Asian Approach to Mental Health. Am J Psychother 30(1): 41-54.

17. Bystritsky A, Stein M, Herman, R (2017) Complementary and alternative treatments for anxiety symptoms and disorders: Physical, cognitive, and spiritual interventions.

18. Zheng G, Liu F, Li S, Huang M, Tao J, et al. (2015) Tai Chi and the protection of cognitive ability: A systematic review of prospective studies in healthy adults. Am J Prev Med 49(1): 89-97.

19. Ergil KV (2015) Traditional medicine of China and East Asia. In: Micozzi MS (Ed), Fundamentals of Complementary and Alternative Medicine, pp. 477-506.

20. Gillespie LD, Robertson MC, Gillespie WJ, Lamb SE, Gates S, et al. (2012) Interventions for preventing falls in older people living in the community. Cochrane Database Syst Rev (9): CD007146.

21. Yin J, Dishman RK (2014) The effect of Tai Chi and Qigong practice on depression and anxiety symptoms: A systematic review and metaregression analysis of randomized controlled trials. Mental Health and Physical Activity 7(3): 135-146.

22. Staples JK, Hamilton MF, Uddo M (2013) A yoga program for the symptoms of post-traumatic stress disorder in veterans. Mil Med 178(8): 854-860.

23. Bower JE, Greendal G, Crosswell ED, Garet D, Sternlieb B, et al. (2014) Yoga reduces inflammatory signaling in fatigued breast cancer survivors: A randomized controlled trial. Psychoneuroendocrinology 43: 20-29.

24. Kawanishi Y, Hanley SJ, Tabata K, Nakagi Y, Yoshioka E, et al. (2015) Effects of prenatal yoga: a systematic review of randomized controlled trials. Nihon Koshu Eisei Zasshi 62(5): 221-231.

25. Orme Johnson D, Barnes V (2014) Effects of the transcendental meditation technique on trait anxiety: A meta-analysis of randomized controlled trials. J Altern Complement Med 20(5): 330-341. 
26. Wachholtz AB, Pargament KI (2005) Is spirituality a critical ingredient of meditation? comparing the effects of spiritual meditation, secular meditation, and relaxation on spiritual, psychological, cardiac, and pain outcomes. J Behav Med 28(4): 369-384.

27. Keng S, Smoski MJ, Robins CJ (2011) Effects of mindfulness on psychological health: A review of empirical studies. Clin Psychol Rev 31(6):1041-1056.

28. Chiesa A, Calati R, Serretti A (2011) Does Mindfulness Training Improve Cognitive Abilities A Systematic Review of Neuropsychological Findings. Clin Psychol Rev 31(3): 449-464.

29. Garrison KA, Zeffiro TA, Scheinost D, Constable RT, Brewer JA (2015) Meditation leads to reduced default mode network activity beyond an active task. Cogn Affect Behav Neurosci 15(3): 712-720.

30. Tang Y, Hölzel BK, Posner MI (2015) The neuroscience of mindfulness meditation. Nat Rev Neurosci 16(4): 213-225.

31. Gard T, Hölzel B, Lazar S (2014) The potential effects of meditation on age-related cognitive decline: A systematic review. Ann N Y Acad Sci 13071(1): 89-103.

32. Loucks EB, Schuman-Olivier Z, Britton WB, Fresco DM, Desbordes G, et al. (2015) Mindfulness and cardiovascular disease risk: State of the evidence, plausible mechanisms, and theoretical framework. Curr Cardiol Rep 17(12): 112.

33. Sharma M, Rush SE (2014) Mindfulness-based stress reduction as a stress management intervention for healthy individuals: A systematic review. J Evid Based Complementary Altern Med 19(4): 271-286.

34. Lamothea M, Rondeaua E, Malboeuf-Hurtubiseb C, Duvala M, Sultana S (2016) Outcomes of MBSR or MBSR-based interventions in health careproviders: A systematic review with a focus on empathy and emotional competencies. Complement Ther Med (24): 19-28.

35. Goyal M, Singh S, Sibinga E, Gould N, Rowland Seymour A, et al. (2014) Meditation Programs for Psychological Stress and Well-being: A Systematic Review and Meta-analysis. JAMA Intern Med 174(3): 357368.
36. (2015) Centers for Disease Control and Prevention. Heart Disease Fact Sheet.

37. Younge JO, Gotink RA, Baena CP, Roos Hesselink JW, Hunink M (2016) Mind-body practices for patients with cardiac disease: a systematic review and meta-analysis. Eur J Prev Cardiol 22(11): 1385-1398.

38. Loucks E, Britton W, Howe C, Eaton C, Buka S (2015b) Positive Associations of Dispositional Mindfulness with Cardiovascular Health: The New England Family Study. Int J Behav Med 22(4): 540-550.

39. Zeidan F, Johnson S, Gordon N, Goolkasian P (2010) Effects of brief and sham mindfulness meditation on mood and cardiovascular variables. J Altern Complement Med 16(8): 867-873.

40. May R, Bamber M, Seibert G, Sanchez Gonzalez M, Leonard J, et al. (2016) Understanding the physiology of mindfulness: Aortic hemodynamics and heart rate variability. Stress 19(2): 168-174.

41. Olex S, Newberg A, Figueredo VM (2014) Meditation: Should a cardiologist care? Int J Cardiol168(3): 1805-1810.

42. Morgan N, Irwin MR, Chung M, Wang C (2014) The effects of mindbody therapies on the immune system: Meta-analysis. PLoS One 9(7): e100903.

43. Kaliman P, Álvarez López MJ, Cosín Tomás M, Rosenkranz MA, Lutz A, et al. (2014) Rapid changes in histone deacetylases and inflammatory gene expression in expert meditators. Psychoneuroendocrinology 40: 96-107.

44. Gao W (2015) Functional Network Development During the First Year: Relative Sequence and Socioeconomic Correlations. Cereb Cortex 25(9): 2919-2928.

45. Ospine MB, Bond TK, Karkhaneh M, Tjosvold L, Vandermeer B, et al. (2007) Meditation Practices for Health: Stae of the Research. Evid Rep Technol Assess (Full Rep) (155): 1-263.

46. Lama D, Vreeland N (2001) An Open Heart: Practicing Compassion in Everyday Life. UK. 